Pacific

Journal of

Mathematics

\title{
A REMARK ON THE NOETHERIAN PROPERTY OF POWER SERIES RINGS
}

Byung Gyun Kang AND Phan Thanh ToAN

Volume $283 \quad$ No. 2

August 2016 


\title{
A REMARK ON THE NOETHERIAN PROPERTY OF POWER SERIES RINGS
}

\author{
Byung Gyun Kang AND Phan Thanh TOAN
}

\begin{abstract}
Let $\alpha$ be a (finite or infinite) cardinal number. An ideal of a ring $R$ is called an $\alpha$-generated ideal if it can be generated by a set with cardinality at most $\alpha$. A ring $R$ is called an $\alpha$-generated ring if every ideal of $R$ is an $\alpha$-generated ideal. When $\alpha$ is finite, the class of $\alpha$-generated rings has been studied in literature by scholars such as I. S. Cohen and R. Gilmer. In this paper, the class of $\alpha$-generated rings when $\alpha$ is infinite (in particular, when $\alpha=\aleph_{0}$, the smallest infinite cardinal number) is considered. Surprisingly, it is proved that the concepts " $\aleph_{0}$-generated ring" and "Noetherian ring" are the same for the power series ring $R \llbracket X \rrbracket$. In other words, if every ideal of $R \llbracket X \rrbracket$ is countably generated, then each of them is in fact finitely generated. This shows a strange behavior of the power series ring $R \llbracket X \rrbracket$ compared to that of the polynomial ring $R[X]$. Indeed, for any infinite cardinal number $\alpha$, it is proved that $R$ is an $\alpha$-generated ring if and only if $R[X]$ is an $\alpha$-generated ring, which is an analogue of the Hilbert basis theorem stating that $R$ is a Noetherian ring if and only if $R[X]$ is a Noetherian ring. Let $O$ be the ring of algebraic integers. Under the continuum hypothesis, we show that $O \llbracket X \rrbracket$ contains an $|\mathcal{O} \llbracket X \rrbracket|$-generated (and hence uncountably generated) ideal which is not a $\beta$-generated ideal for any cardinal number $\beta<|O\|X\||$ and that the concepts " $\aleph_{1}$-generated ring" and " $\aleph_{0}$-generated ring" are different for the power series ring $R \llbracket X \rrbracket$.
\end{abstract}

\section{Introduction}

In this paper, a ring means a commutative ring with identity. Let $R$ be a ring and let $n$ be a positive integer. An ideal $I$ of $R$ is called an $n$-generated ideal if $I$ can be generated by a set with cardinality $\leq n$. We call $R$ an $n$-generated ring if every ideal of $R$ is an $n$-generated ideal. This class of $n$-generated rings was first introduced and studied by Cohen [1950]. Principal ideal rings are obviously 1-generated rings. It is well known that Dedekind domains are 2-generated rings. For each

Phan Thanh Toan is the corresponding author.

MSC2010: 03E10, 13A15, 13B25, 13E05, 13F25.

Keywords: countably generated ideal, $n$-generated ideal, Noetherian ring, polynomial ring, power series ring. 
integer $n \geq 2$, Matson [2009] gave an example of an $n$-generated ring which is not an $(n-1)$-generated ring. Cohen [1950] proved that if $D$ is an $n$-generated integral domain, then the Krull dimension of $D$ is at most 1 . An easy proof of this result was later given by Gilmer [1973]. He further showed [1972b] that the result is valid for $n$-generated rings (see also [Sally 1978, Theorem 1.2, p. 51]). By definition, every $n$-generated ring is a Noetherian ring. However, the converse does not hold [Cohen 1950]. Gilmer [1973] proved a nice result that if $R$ is an $n$-generated ring, then the integral closure of $R$ is a Noetherian Prüfer ring. He also showed that a Noetherian ring $R$ is an $n$-generated ring (for some $n$ ) if and only if there exists a positive integer $m$ such that $R_{M}$ is an $m$-generated ring for each maximal ideal $M$ of $R$ [Gilmer 1972a; 1972b]. For more on $n$-generated ideals and rings, we refer the readers to [Ameziane Hassani et al. 1996; Cohen 1950; Gilmer 1972b; 1973; Heinzer and Lantz 1983; Matsuda 1979; McLean 1982; Okon et al. 1992; Okon and Vicknair 1992; 1993; Rush 1991; 1992; Sally 1978; Shalev 1986].

According to Cohen and Gilmer's results, the class of $n$-generated rings is rather small. It is a subclass of Noetherian rings with Krull dimension at most 1 . We generalize the definition of $n$-generated rings in a natural way as follows. Let $\alpha$ be a (finite or infinite) cardinal number (e.g., $\alpha=1,2, \ldots, \aleph_{0}, \aleph_{1}, \ldots$ ). An ideal $I$ of a ring $R$ is called an $\alpha$-generated ideal if $I$ can be generated by a set with cardinality at most $\alpha . R$ is called an $\alpha$-generated ring if every ideal of $R$ is an $\alpha$-generated ideal. In this paper, we mainly deal with the class of $\alpha$-generated rings when $\alpha=\aleph_{0}$, the smallest infinite cardinal number. By definition, an $\aleph_{0}$-generated ring is a ring whose ideals are countably generated. Trivial examples of $\aleph_{0}$-generated rings are those that have only countably many elements (so that each ideal has itself as a countable generating set). Every Noetherian ring is obviously an $\aleph_{0}$-generated ring. However, the converse does not hold. Polynomial rings $R\left[X_{1}, X_{2}, \ldots, X_{n}, \ldots\right]$ in countably infinite indeterminates over countable rings $R$, the ring 0 of algebraic integers, the ring $\operatorname{Int}(\mathbb{Z})$ of integer-valued polynomials on $\mathbb{Z}$, and 1-dimensional nondiscrete valuation domains are good examples of $\aleph_{0}$-generated rings that are not Noetherian rings.

Even though the class of $\aleph_{0}$-generated rings is strictly larger than the class of Noetherian rings, we show that, when restricted to power series rings, they are actually the same. In other words, the concepts " $\aleph_{0}$-generated ring" and "Noetherian ring" are the same for the power series ring $R \llbracket X \rrbracket$ (Theorem 13). This means if every ideal of $R \llbracket X \rrbracket$ is countably generated, then each of them is in fact finitely generated. This shows a strange behavior of the power series ring $R \llbracket X \rrbracket$ compared to that of the polynomial ring $R[X]$. Indeed, for any infinite cardinal number $\alpha$, we prove that $R$ is an $\alpha$-generated ring if and only if $R[X]$ is an $\alpha$-generated ring (Theorem 22), which is an analogue of the Hilbert basis theorem stating that $R$ is a Noetherian ring if and only if $R[X]$ is a Noetherian ring. We show under 
the continuum hypothesis that (1) $\mathcal{O} \llbracket X \rrbracket$ contains an $|\mathcal{O} \llbracket X \rrbracket|$-generated (and hence uncountably generated) ideal which is not a $\beta$-generated ideal for any cardinal number $\beta<|\mathcal{O} \llbracket X \rrbracket|$ (Corollary 16) and that (2) $\mathcal{O} \llbracket X \rrbracket$ is an $\aleph_{1}$-generated ring that is not an $\aleph_{0}$-generated ring (Corollary 17 ). In fact, these two results hold if 0 is replaced by any non-Noetherian countable ring $R$. As a consequence, it is shown that the concepts " $\aleph_{1}$-generated ring" and " $\aleph_{0}$-generated ring" are different (while the concepts " $\aleph_{0}$-generated ring" and "Noetherian ring" are the same) for the power series ring $R \llbracket X \rrbracket$.

\section{Some examples of $\alpha$-generated rings}

For each integer $n \geq 2$, Matson [2009] gave an example of an $n$-generated ring which is not an $(n-1)$-generated ring. For an infinite cardinal number $\alpha$, we give an example of an $\alpha$-generated ring that is not a $\beta$-generated ring for any cardinal number $\beta<\alpha$.

Proposition 1. For an infinite cardinal number $\alpha$, there exists an $\alpha$-generated ring that is not a $\beta$-generated ring for any cardinal number $\beta<\alpha$.

Proof. Let $R$ be any ring with cardinality $<\alpha$ and let $\left\{X_{\lambda}\right\}_{\lambda \in \Lambda}$ be a set of indeterminates over $R$, where $\Lambda$ is a set of cardinality $\alpha$. Then the polynomial ring $R\left[\left\{X_{\lambda}\right\}_{\lambda \in \Lambda}\right]$ is clearly an $\alpha$-generated ring since it has cardinality $\alpha$. We now show that the ideal $J$ of $R\left[\left\{X_{\lambda}\right\}_{\lambda \in \Lambda}\right]$ generated by $\left\{X_{\lambda}\right\}_{\lambda \in \Lambda}$ is not a $\beta$-generated ideal for any cardinal number $\beta<\alpha$. Let $\beta$ be any cardinal number such that $\beta<\alpha$. If $J$ is a $\beta$-generated ideal, then $J=\left(\left\{f_{\mu}\right\}\right)$ for some $f_{\mu} \in J$ such that $\left|\left\{f_{\mu}\right\}\right| \leq \beta$. Since each $f_{\mu}$ involves only finitely many indeterminates, $\left(\left\{X_{\lambda}\right\}_{\lambda \in \Lambda}\right)=J=\left(\left\{f_{\mu}\right\}\right) \subseteq\left(\left\{X_{\lambda}\right\}_{\lambda \in \Gamma}\right)$ for some subset $\Gamma$ of $\Lambda$ such that $|\Gamma|<|\Lambda|=\alpha$, a contradiction.

In the next section, we are going to prove that the concepts " $\aleph_{0}$-generated ring" and "Noetherian ring" are the same for the power series ring $R \llbracket X \rrbracket$. We however note that these two concepts are different in general. We give here some examples of (finite-dimensional or infinite-dimensional) $\aleph_{0}$-generated rings that are not Noetherian rings.

Example 2. Let $R_{1}:=R\left[X_{1}, X_{2}, \ldots, X_{n}, \ldots\right]$ be the polynomial ring in countably infinite indeterminates over a countable ring $R$. Then $R_{1}$ is an $\aleph_{0}$-generated ring since it is countable. It is easy to see that the ideal of $R_{1}$ generated by $X_{1}, X_{2}, \ldots, X_{n}, \ldots$ is not a finitely generated ideal and hence $R_{1}$ is not a Noetherian ring.

In Example 2, the (Krull) dimension of $R_{1}$ is infinite. We now give examples of finite-dimensional $\aleph_{0}$-generated rings that are not Noetherian rings.

Example 3. Let $\mathcal{O}$ be the ring of algebraic integers (an algebraic integer is a complex number that is integral over $\mathbb{Z}$ ). It is well known that 0 is a 1-dimensional 
non-Noetherian Bézout domain (for example, see p. 72 of [Kaplansky 1974]). However, since $\mathbb{O}$ is countable, it is an $\aleph_{0}$-generated ring.

Example 4. Let $\operatorname{Int}(\mathbb{Z})$ be the ring of integer-valued polynomials on $\mathbb{Z}$, i.e.,

$$
\operatorname{Int}(\mathbb{Z}):=\{f \in \mathbb{Q}[X] \mid f(\mathbb{Z}) \subseteq \mathbb{Z}\} .
$$

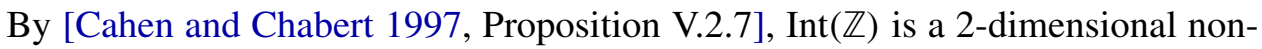
Noetherian domain. However, since $\operatorname{Int}(\mathbb{Z})$ is countable, it is an $\aleph_{0}$-generated ring.

So far, the given examples of $\aleph_{0}$-generated rings are all countable. We finally show that there do exist uncountable $\aleph_{0}$-generated rings that are not Noetherian rings (see Example 6).

Proposition 5. If $V$ is a 1-dimensional nondiscrete valuation domain, then $V$ is an $\aleph_{0}$-generated ring that is not a Noetherian ring.

Proof. Since $V$ is a 1-dimensional valuation domain, its value group is (isomorphic to) a subgroup of $\mathbb{R}$, the (additive) group of real numbers. This implies that every ideal of $V$ is countably generated, i.e., $V$ is an $\aleph_{0}$-generated ring. Since $V$ is nondiscrete, it is not a Noetherian ring.

Example 6. Let $K$ be a field and let $V$ be the valuation ring of the field $K(X ; \mathbb{R})$, which is the quotient field of the group ring $K[X ; \mathbb{R}]$ of $\mathbb{R}$ over $K$, associated with the valuation $v$ defined by

$$
v\left(\sum_{i=0}^{n} a_{r_{i}} X^{r_{i}}\right):=\min \left\{r_{i} \mid i=0,1, \ldots, n\right\} .
$$

Then $V$ is a 1-dimensional nondiscrete valuation domain with value group $\mathbb{R}$. Hence, $V$ is an $\aleph_{0}$-generated ring that is not a Noetherian ring by Proposition 5 . Since $\mathbb{R}$ is uncountable, so is $V$.

\section{Power series rings over $\alpha$-generated rings}

In this section, we prove that the power series ring $R \llbracket X \rrbracket$ is an $\aleph_{0}$-generated ring if and only if $R \llbracket X \rrbracket$ is a Noetherian ring (and hence) if and only if $R$ is a Noetherian ring. In order to prove this result, we only need to show that if $R$ is a non-Noetherian ring, then the power series ring $R \llbracket X \rrbracket$ is not an $\aleph_{0}$-generated ring since, if $R$ is a Noetherian ring, then $R \llbracket X \rrbracket$ is also a Noetherian ring (for example, see [Kaplansky 1974, Theorem 71]) and hence an $\aleph_{0}$-generated ring.

Suppose that $R$ is a non-Noetherian ring. Our task is to construct an ideal $J$ of $R \llbracket X \rrbracket$ that cannot be generated by any countable subset of $J$. The desired uncountable generating set for $J$ is indexed by the following special uncountable set, which is called a fathomless set. 
3.1. Fathomless sets. Let $\mathbb{N}=\{1,2, \ldots\}$ be the set of positive integers, and let $\mathcal{U}$ be the set of all subsets $U$ of $\mathbb{N}$ such that $U=\{n, n+1, \ldots\}$ for some $n \in \mathbb{N}$. For two strictly increasing sequences $s=\left\{s_{n}\right\}$ and $t=\left\{t_{n}\right\}$ of positive integers, we set $s \gg t$ (we also write $t \ll s$ ) if for each positive integer $k$ there is a set $U \in \mathcal{U}$ (depending on $k$ ) such that $s_{n}>k t_{n}$ for each $n \in U$, i.e., $s_{n}>k t_{n}$ for all large $n$.

Let $\mathscr{Y}$ be the collection of all $\mathscr{A}$ having the following properties:

(1) $\mathscr{A}$ is a nonempty collection of strictly increasing sequences $s=\left\{s_{n}\right\}$ of positive integers.

(2) If $s \in \mathscr{A}$ then $s \gg b$, where $b$ is the sequence defined by $b_{n}:=n$ for all $n$.

(3) If $s, t \in \mathscr{A}$ and $s \neq t$, then $s \gg t$ or $t \gg s$.

If $u$ is the sequence defined by $u_{n}:=b_{n}^{2}$ for each $n$, then it is easy to see that $u$ is a strictly increasing sequence of positive integers and that $u \gg b$. It follows that the set $\mathscr{Y}$ is nonempty. We order $\mathscr{Y}$ by set-theoretic inclusion. By Zorn's lemma, there exists a maximal element in $\mathscr{Y}$. Let $\mathscr{A}$ be a maximal element in $\mathscr{Y}$. This choice of $\mathscr{A}$ will be fixed through the rest of the article. For $s, t \in \mathscr{A}$, we define $s \lll t$ if and only if $s=t$ or $s \ll t$. Then $(\mathscr{A}, \ll)$ becomes a totally ordered set.

Definition 7. A totally ordered set $(\mathscr{Y}, \ll)$ is called a fathomless set if, for every nonempty countable subset $\mathscr{C}$ of $\mathscr{Y}$, there exists an element $y \in \mathscr{Y}$ such that $y \ll \mathscr{C}$, i.e., $y \ll c$ for all $c \in \mathscr{C}$.

The following theorem tells us that the set $(\mathscr{A}, \ll)$ is a fathomless set (for the proof, see [Kang et al. 2013, Theorem 5]).

Theorem 8. The set $(\mathscr{A}, \ll)$ is a fathomless set.

Remark 9. By definition, every fathomless set is an uncountable set. Hence, the set $(\mathscr{A}, \ll)$ is uncountable.

3.2. Power series rings over $\aleph_{0}$-generated rings. Using the above fathomless set $(\mathscr{A}, \ll)$, we construct generators for an ideal $J$ of $R \llbracket X \rrbracket$ that is not countably generated. The following observation is useful:

Proposition 10. For a ring $R$, the following are equivalent:

(1) $R$ is a non-Noetherian ring.

(2) There exists a sequence $a_{0}, a_{1}, \ldots, a_{m}, \ldots$ of elements in $R$ such that

$$
a_{m} \notin\left(a_{0}, a_{1}, \ldots, a_{m-1}\right)
$$

for each $m \geq 1$.

Proof. (1) $\Longrightarrow$ (2) Suppose that $R$ is a non-Noetherian ring. Then there exists an ideal $I$ of $R$ such that $I$ is not finitely generated. We will find the desired sequence $a_{0}, a_{1}, \ldots, a_{m}, \ldots$ of elements in $R$ by using induction as follows. Choose an 
element $a_{0} \in I$. Since $\left(a_{0}\right) \subsetneq I$, there exists an element $a_{1} \in I \backslash\left(a_{0}\right)$. Suppose that there exist elements $a_{0}, a_{1}, \ldots, a_{m-1}$ in $I(m \geq 2)$ such that $a_{i} \notin\left(a_{0}, a_{1}, \ldots, a_{i-1}\right)$ for each $1 \leq i \leq m-1$. Since $\left(a_{0}, a_{1}, \ldots, a_{m-1}\right) \subsetneq I$, there exists an element $a_{m} \in I \backslash\left(a_{0}, a_{1}, \ldots, a_{m-1}\right)$.

(2) $\Longrightarrow(1)$ The ideal $\left(a_{0}, a_{1}, \ldots, a_{m}, \ldots\right)$ of $R$ is not finitely generated.

Let $R$ be a non-Noetherian ring. By Proposition 10, there exists a sequence $a_{0}, a_{1}, \ldots, a_{m}, \ldots$ of elements in $R$ such that

$$
a_{m} \notin\left(a_{0}, a_{1}, \ldots, a_{m-1}\right)
$$

for each $m \geq 1$. For each integer $m \geq 0$, we let $I_{m}:=\left(a_{0}, a_{1}, \ldots, a_{m}\right)$. Then $a_{m} \notin I_{m-1}$ for each $m \geq 1$. For each sequence $s=\left\{s_{n}\right\} \in \mathscr{A}$, we define

$$
f_{s}:=a_{0}+a_{1} X^{s_{1}}+a_{2} X^{s_{2}}+\cdots+a_{n} X^{s_{n}}+\cdots \in R \llbracket X \rrbracket .
$$

We let $J$ be the ideal of $R \llbracket X \rrbracket$ generated by all $f_{s}$ with $s \in \mathscr{A}$.

Remark 11. The generators $f_{s}$ of $J$ are constructed by stretching out the coefficients of the power series $\sum_{n=0}^{\infty} a_{n} X^{n}$ so that its coefficient at $X^{s_{n}}$ ( $s_{n}$ is much greater than $n$ for all large $n$ since $s \gg b$ ) is still $a_{n}$. This property will play a crucial role in showing that $J$ is not a countably generated ideal.

An ideal $I$ of a ring $R$ is called an uncountably generated ideal if it is not a countably generated ideal. If $R$ is a non-Noetherian ring, then so is the power series ring $R \llbracket X \rrbracket$. Hence, $R \llbracket X \rrbracket$ has some ideals that are not finitely generated. These ideals can be either countably generated or uncountably generated. However, we show that $R \llbracket X \rrbracket$ has at least one uncountably generated ideal if $R$ is a nonNoetherian ring.

Theorem 12. If $R$ is a non-Noetherian ring, then the power series ring $R \llbracket X \rrbracket$ has at least one uncountably generated ideal.

Proof. It suffices to show that the ideal $J$ constructed above is not a countably generated ideal. Suppose on the contrary that $J$ is countably generated. Then there exists a countable subset $\mathscr{B}$ of $\mathscr{A}$ such that $J$ is generated by $\left\{f_{s} \mid s \in \mathscr{B}\right\}$. Since $\mathscr{A}$ is a fathomless set, there exists a sequence $v \in \mathscr{A}$ such that $v \ll \mathscr{B}$. Since $f_{v} \in J$, $f_{v}$ is a finite sum of elements of the form $h(s) f_{s}$,

$$
f_{v}=\sum_{s} h(s) f_{s},
$$

where $h(s) \in R \llbracket X \rrbracket$ and $s \in \mathscr{B}$. Since $v \ll \mathscr{B}$, by taking a finite intersection of members of $U$, we can find a set $U \in U$ such that $v_{m}<s_{m}$ for each $m \in U$ and for each $s$ appearing in the finite sum (1). Choose any number $m \in U$. Since $v_{m}<s_{m}$, the coefficient of $f_{s}$ at $X^{j}$ belongs to $I_{m-1}$ for all $j \leq v_{m}$. It follows that the 
coefficient of $h(s) f_{s}$ at $X^{v_{m}}$ belongs to $I_{m-1}$. This holds for every $s$ appearing in the finite sum (1). Therefore, the coefficient of $\sum_{s} h(s) f_{s}$ at $X^{v_{m}}$ belongs to $I_{m-1}$. This is a contradiction since the coefficient of $f_{v}=\sum_{s} h(s) f_{s}$ at $X^{v_{m}}$ is $a_{m}$ and $a_{m} \notin I_{m-1}$.

We can now obtain the main result of the paper.

Theorem 13. For a ring $R$, the following are equivalent:

(1) $R \llbracket X \rrbracket$ is an $\aleph_{0}$-generated ring.

(2) $R \llbracket X \rrbracket$ is a Noetherian ring.

(3) $R$ is a Noetherian ring.

Proof. We only need to prove that (1) implies (3). However, this follows from Theorem 12.

Since a ring $R$ is a Noetherian ring if and only if every countably generated ideal is finitely generated, we have the following corollary.

Corollary 14. For a ring $R$, the following are equivalent:

(1) Every ideal of $R \llbracket X \rrbracket$ is countably generated.

(2) Every countably generated ideal of $R \llbracket X \rrbracket$ is finitely generated.

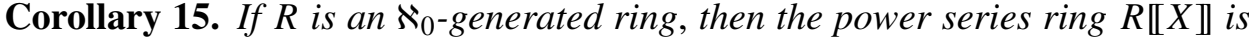
not necessarily an $\aleph_{0}$-generated ring.

Proof. Let $R$ be any $\aleph_{0}$-generated ring which is not a Noetherian ring (see Proposition 5 and Examples 2, 3, and 4). Then $R \llbracket X \rrbracket$ is not an $\aleph_{0}$-generated ring by Theorem 13 .

Corollary 16. Let 0 be the ring of algebraic integers and let $\alpha=|\mathcal{O} \llbracket X \rrbracket|$. Then, under the continuum hypothesis, $\mathcal{O} \llbracket X \rrbracket$ contains an $\alpha$-generated ideal that is not a $\beta$-generated ideal for any cardinal number $\beta<\alpha$.

Proof. We have $\alpha=|\mathcal{O} \llbracket X \rrbracket|=2^{\aleph_{0}}=\aleph_{1}$ under the continuum hypothesis. By Theorem 12, $0 \llbracket X \rrbracket$ has an uncountably generated ideal $J$. Obviously, $J$ is an $\alpha$-generated ideal since $|J| \leq|O \llbracket X \rrbracket|=\alpha$. But, since $J$ is not countably generated, it is not a $\beta$-generated ideal for any cardinal number $\beta<\aleph_{1}=\alpha$.

The following corollary shows that the concepts " $\aleph_{1}$-generated ring" and " $\aleph_{0}$ generated ring" are different for the power series ring $R \llbracket X \rrbracket$.

Corollary 17. Under the continuum hypothesis, $O \llbracket X \rrbracket$ is an $\aleph_{1}$-generated ring but not an $\aleph_{0}$-generated ring.

Proof. Since $|O \llbracket X \rrbracket|=\aleph_{1}, O \llbracket X \rrbracket$ is an $\aleph_{1}$-generated ring. The fact that $O \llbracket X \rrbracket$ is not an $\aleph_{0}$-generated ring follows from Corollary 16. 
Remark 18. Corollaries 16 and 17 hold for any non-Noetherian countable ring (see Examples 2, 3, and 4).

In the rest of this section, we consider power series rings over $n$-generated rings, where $n$ is a positive integer. We first note that, for the power series ring $R \llbracket X \rrbracket$ to be an $n$-generated ring, it is necessary that the ring $R$ be zero-dimensional.

Proposition 19. If the power series ring $R \llbracket X \rrbracket$ is an n-generated ring for some positive integer $n$, then $\operatorname{dim} R=0$.

Proof. If $R \llbracket X \rrbracket$ is an $n$-generated ring, then $\operatorname{dim} R \llbracket X \rrbracket \leq 1$. It is easy to see that $\operatorname{dim} R+1 \leq \operatorname{dim} R \llbracket X \rrbracket$. Thus, $\operatorname{dim} R+1 \leq \operatorname{dim} R \llbracket X \rrbracket \leq 1$ and hence $\operatorname{dim} R=0$.

Proposition 20. Suppose that $D$ is an integral domain. Then the following are equivalent:

(1) $D \llbracket X \rrbracket$ is an n-generated ring for some positive integer $n$.

(2) $D \llbracket X \rrbracket$ is a 1-generated ring.

(3) $D$ is a field.

Proof. (3) $\Longrightarrow(2) \Longrightarrow$ (1) These are obvious.

$(1) \Longrightarrow$ (3) By Proposition $19, \operatorname{dim} D=0$. Thus, $D$ is a field by the assumption that $D$ is an integral domain.

Remark 21. By Proposition 20, if $D$ is an (n-generated) integral domain which is not a field, then the power series ring $D \llbracket X \rrbracket$ is never an $m$-generated ring for any positive integer $m$. In particular, $D \llbracket X \rrbracket$ has an ideal that is not an $(n+1)$-generated ideal despite the fact that all prime ideals of $D \llbracket X \rrbracket$ are $(n+1)$-generated ideals (see [Kaplansky 1974, Theorem 70]).

\section{Polynomial rings over $\alpha$-generated rings}

In this section, we prove that for any infinite cardinal number $\alpha$, a ring $R$ is an $\alpha$ generated ring if and only if the polynomial ring $R[X]$ is an $\alpha$-generated ring, which is an analogue of the Hilbert basis theorem, which states that $R$ is a Noetherian ring if and only if $R[X]$ is a Noetherian ring. We also note that the result fails if $\alpha$ is a finite cardinal number.

Theorem 22. For any infinite cardinal number $\alpha$, a ring $R$ is an $\alpha$-generated ring if and only if the polynomial ring $R[X]$ is an $\alpha$-generated ring.

Proof. We follow the standard proof of the Hilbert basis theorem. Any homomorphic image of an $\alpha$-generated ring is obviously an $\alpha$-generated ring. Hence, if $R[X]$ is an $\alpha$-generated ring, then so is $R$.

For the converse, suppose that $R$ is an $\alpha$-generated ring and let $J$ be an ideal of $R[X]$. We show that $J$ is an $\alpha$-generated ideal. For each $n \geq 0$, let $I_{n}$ be the 
set of $r \in R$ such that $r=0$ or $r$ is the leading coefficient of a polynomial in $J$ of degree $n$. For each $n \geq 0$, since $R$ is an $\alpha$-generated ring, $I_{n}$ is an $\alpha$-generated ideal. Let

$$
\left\{r_{n \lambda} \mid \lambda \in \Lambda_{n}\right\}
$$

be a generating set of $I_{n}$ with cardinality at most $\alpha$. For each $r_{n \lambda}$, let $f_{n \lambda} \in J$ be a polynomial of degree $n$ with leading coefficient $r_{n \lambda}$. We will show that the ideal $J$ of $R[X]$ is generated by the set

$$
F:=\left\{f_{n \lambda} \mid n \geq 0, \lambda \in \Lambda_{n}\right\},
$$

which also has cardinality at most $\alpha$. Denote by $(F)$ the ideal of $R[X]$ generated by $F$. Since $F \subseteq J$, we have $(F) \subseteq J$. Conversely, the polynomials of degree 0 in $J$ are precisely the elements of $I_{0}$ and hence are contained in $(F)$. Proceeding by induction, assume that $(F)$ contains all polynomials of $J$ of degree less than $k$ and let $g \in J$ have degree $k$ and leading coefficient $r$. Then

$$
r=\sum_{i=1}^{m} s_{i} r_{k \lambda_{i}}
$$

for some $s_{i} \in R$ and $\lambda_{i} \in \Lambda_{k}$. The polynomial $\sum_{i=1}^{m} s_{i} f_{k \lambda_{i}}$ also has degree $k$ and leading coefficient $r$. Hence,

$$
g-\sum_{i=1}^{m} s_{i} f_{k \lambda_{i}} \in J
$$

has degree at most $k-1$. By the induction hypothesis,

$$
g-\sum_{i=1}^{m} s_{i} f_{k \lambda_{i}} \in(F)
$$

and hence $g \in(F)$. Therefore, $J=(F)$ is an $\alpha$-generated ideal.

Proposition 23. If the polynomial ring $R[X]$ is an $n$-generated ring for some positive integer $n$, then $\operatorname{dim} R=0$.

Proof. As in the proof of Proposition 19, we have $\operatorname{dim} R+1 \leq \operatorname{dim} R[X] \leq 1$ and hence $\operatorname{dim} R=0$.

As in the power series ring case, we can prove the following:

Proposition 24. Suppose that $D$ is an integral domain. Then the following are equivalent:

(1) $D[X]$ is an n-generated ring for some positive integer $n$.

(2) $D[X]$ is a 1-generated ring.

(3) D is a field. 
Remark 25. By Proposition 24, if $D$ is an (n-generated) integral domain which is not a field, then the polynomial ring $D[X]$ is never an $m$-generated ring for any positive integer $m$. In particular, Theorem 22 always fails for any finite cardinal number $\alpha$.

Remarks 26. (1) For rings with zero-divisors, the polynomial ring $R[X]$ may not be a 2 -generated ring even if the ring $R$ is a 1-generated ring. For example, let $R=V /\left(a^{3}\right)$, where $V$ is a 1-dimensional discrete valuation domain (or equivalently, a local principal ideal domain) with maximal ideal $M=(a)$. Then $R$ is a 1-generated ring. However, if $\bar{M}$ denotes the maximal ideal $M /\left(a^{3}\right)$ of $R$, then $\bar{M}^{2} \neq 0$. By [Matsuda 1979, (5.7)], $R[X]$ is not a 2-generated ring.

(2) More generally, for any integer $n \geq 2$, let $R=V /\left(a^{n+1}\right)$, where $V$ is the same as above. Then $R$ is a 1-generated ring. However, by [Matsuda 1979, (5.13)], the polynomial ring $R[X]$ is not an $n$-generated ring.

\section{Acknowledgements}

The authors would like to thank the referee for his/her helpful comments and suggestions. This research was supported by Basic Science Research Program through the National Research Foundation of Korea (NRF) funded by the Ministry of Education (2013R1A1A2020783).

\section{References}

[Ameziane Hassani et al. 1996] S. Ameziane Hassani, M. Fontana, and S.-E. Kabbaj, "Group rings $R[G]$ with 3-generated ideals when $R$ is Artinian", Comm. Algebra 24:4 (1996), 1253-1280. MR 1380596

[Cahen and Chabert 1997] P.-J. Cahen and J.-L. Chabert, Integer-valued polynomials, Mathematical Surveys and Monographs 48, American Mathematical Society, Providence, RI, 1997. MR 1421321

[Cohen 1950] I. S. Cohen, "Commutative rings with restricted minimum condition", Duke Math. J. 17 (1950), 27-42. MR 0033276

[Gilmer 1972a] R. Gilmer, Multiplicative ideal theory, Pure and Applied Mathematics 12, Dekker, New York, 1972. MR 0427289

[Gilmer 1972b] R. Gilmer, "On commutative rings of finite rank", Duke Math. J. 39 (1972), 381-383. MR 0292810

[Gilmer 1973] R. Gilmer, "The n-generator property for commutative rings", Proc. Amer. Math. Soc. 38 (1973), 477-482. MR 0309922

[Heinzer and Lantz 1983] W. Heinzer and D. Lantz, "Commutative rings with acc on $n$-generated ideals”, J. Algebra 80:1 (1983), 261-278. MR 690717

[Kang et al. 2013] B. G. Kang, K. A. Loper, T. G. Lucas, M. H. Park, and P. T. Toan, "The Krull dimension of power series rings over non-SFT rings", J. Pure Appl. Algebra 217:2 (2013), 254-258. MR 2969250

[Kaplansky 1974] I. Kaplansky, Commutative rings, revised ed., The University of Chicago Press, 1974. MR 0345945 
[Matson 2009] A. Matson, "Rings of finite rank and finitely generated ideals", J. Commut. Algebra 1:3 (2009), 537-546. MR 2524865

[Matsuda 1979] R. Matsuda, "Torsion-free abelian semigroup rings, V”, Bull. Fac. Sci. Ibaraki Univ. Ser. A 11 (1979), 1-37. MR 536898

[McLean 1982] K. R. McLean, "Local rings with bounded ideals", J. Algebra 74:2 (1982), 328-332. MR 647243

[Okon and Vicknair 1992] J. S. Okon and J. P. Vicknair, "Group rings with $n$-generated ideals", Comm. Algebra 20:1 (1992), 189-217. MR 1145332

[Okon and Vicknair 1993] J. S. Okon and J. P. Vicknair, "One-dimensional monoid rings with n-generated ideals”, Canad. Math. Bull. 36:3 (1993), 344-350. MR 1245819

[Okon et al. 1992] J. S. Okon, D. E. Rush, and P. Vicknair, "Commutative semigroup rings with two-generated ideals", J. London Math. Soc. (2) 45:3 (1992), 417-432. MR 1180253

[Rush 1991] D. E. Rush, "Rings with two-generated ideals", J. Pure Appl. Algebra 73:3 (1991), 257-275. MR 1124788

[Rush 1992] D. E. Rush, “Artin rings with two-generated ideals”, Canad. Math. Bull. 35:1 (1992), 133-135. MR 1157475

[Sally 1978] J. D. Sally, Numbers of generators of ideals in local rings, Dekker, New York, 1978. MR 0485852

[Shalev 1986] A. Shalev, "On the number of generators of ideals in local rings", Adv. in Math. 59:1 (1986), 82-94. MR 825089

Received November 29, 2015. Revised January 15, 2016.

\section{BYUNG GYUN KANG}

DEPARTMENT OF MATHEMATICS

Pohang University of SCIENCE AND TeChNology

POHANG 37673

SOUTH KOREA

bgkang@postech.ac.kr

PHAN THANH TOAN

Division of COMPUTATIONAL Mathematics AND ENGINEERING

INSTITUTE FOR COMPUTATIONAL SCIENCE

TON DUC THANG UNIVERSITY

Ho CHI MINH CITY

VIETNAM

and

FACUlTy OF MATHEMATICS AND STATISTICS

TON DUC THANG UNIVERSITY

Ho Chi Minh CiTY

VIETNAM

phanthanhtoan@tdt.edu.vn 


\title{
PACIFIC JOURNAL OF MATHEMATICS
}

Founded in 1951 by E. F. Beckenbach (1906-1982) and F. Wolf (1904-1989)

$$
\text { msp.org/pjm }
$$

\section{EDITORS}

\author{
Don Blasius (Managing Editor) \\ Department of Mathematics \\ University of California \\ Los Angeles, CA 90095-1555 \\ blasius@math.ucla.edu
}

\author{
Paul Balmer \\ Department of Mathematics \\ University of California \\ Los Angeles, CA 90095-1555 \\ balmer@math.ucla.edu \\ Robert Finn \\ Department of Mathematics \\ Stanford University \\ Stanford, CA 94305-2125 \\ finn@math.stanford.edu \\ Sorin Popa \\ Department of Mathematics \\ University of California \\ Los Angeles, CA 90095-1555 \\ popa@math.ucla.edu
}

\author{
Vyjayanthi Chari \\ Department of Mathematics \\ University of California \\ Riverside, CA 92521-0135 \\ chari@math.ucr.edu \\ Kefeng Liu \\ Department of Mathematics \\ University of California \\ Los Angeles, CA 90095-1555 \\ liu@math.ucla.edu \\ Igor Pak \\ Department of Mathematics \\ University of California \\ Los Angeles, CA 90095-1555 \\ pak.pjm@gmail.com \\ Paul Yang \\ Department of Mathematics \\ Princeton University \\ Princeton NJ 08544-1000 \\ yang@math.princeton.edu
}

\section{PRODUCTION}

Silvio Levy, Scientific Editor, production@msp.org

\section{SUPPORTING INSTITUTIONS}

ACADEMIA SINICA, TAIPEI

CALIFORNIA INST. OF TECHNOLOGY

STANFORD UNIVERSITY

UNIV. OF BRITISH COLUMBIA

UNIV. OF CALIFORNIA, BERKELEY

UNIV. OF CALIFORNIA, DAVIS

UNIV. OF CALIFORNIA, LOS ANGELES

UNIV. OF CALIFORNIA, RIVERSIDE

UNIV. OF CALIFORNIA, SAN DIEGO

UNIV. OF CALIF., SANTA BARBARA
KEIO UNIVERSITY

MATH. SCIENCES RESEARCH INSTITUTE

NEW MEXICO STATE UNIV.

OREGON STATE UNIV.
Daryl Cooper

Department of Mathematics

University of California

Santa Barbara, CA 93106-3080 cooper@math.ucsb.edu

Jiang-Hua Lu

Department of Mathematics

The University of Hong Kong

Pokfulam Rd., Hong Kong

jhlu@maths.hku.hk

$$
\text { Jie Qing }
$$

Department of Mathematics

University of California

Santa Cruz, CA 95064

qing@ cats.ucsc.edu

\author{
UNIV. OF CALIF., SANTA CRUZ \\ UNIV. OF MONTANA \\ UNIV. OF OREGON \\ UNIV. OF SOUTHERN CALIFORNIA \\ UNIV. OF UTAH \\ UNIV. OF WASHINGTON \\ WASHINGTON STATE UNIVERSITY
}

These supporting institutions contribute to the cost of publication of this Journal, but they are not owners or publishers and have no responsibility for its contents or policies.

See inside back cover or msp.org/pjm for submission instructions.

The subscription price for 2016 is US $\$ 440 /$ year for the electronic version, and \$600/year for print and electronic.

Subscriptions, requests for back issues and changes of subscriber address should be sent to Pacific Journal of Mathematics, P.O. Box 4163, Berkeley, CA 94704-0163, U.S.A. The Pacific Journal of Mathematics is indexed by Mathematical Reviews, Zentralblatt MATH, PASCAL CNRS Index, Referativnyi Zhurnal, Current Mathematical Publications and Web of Knowledge (Science Citation Index).

The Pacific Journal of Mathematics (ISSN 0030-8730) at the University of California, c/o Department of Mathematics, 798 Evans Hall \#3840, Berkeley, CA 94720-3840, is published twelve times a year. Periodical rate postage paid at Berkeley, CA 94704, and additional mailing offices. POSTMASTER: send address changes to Pacific Journal of Mathematics, P.O. Box 4163, Berkeley, CA 94704-0163.

PJM peer review and production are managed by EditFLOW ${ }^{\circledR}$ from Mathematical Sciences Publishers.

PUBLISHED BY

\section{I. mathematical sciences publishers}

nonprofit scientific publishing

http://msp.org/

(C) 2016 Mathematical Sciences Publishers 


\section{PACIFIC JOURNAL OF MATHEMATICS}

Volume $283 \quad$ No. $2 \quad$ August 2016

The fundamental theorem of tropical differential algebraic geometry

FUENSANTA AROCA, CRISTHIAN GARAY and ZEINAB TOGHANI

A simple solution to the word problem for virtual braid groups

271

PaOlo Bellingeri, Bruno A. Cisneros de la CruZ and Luis

PARIS

Completely contractive projections on operator algebras

DAVID P. BLECHER and MATTHEW NEAL

Invariants of some compactified Picard modular surfaces and applications

AMIR DŽAMBIĆ

Radial limits of bounded nonparametric prescribed mean curvature surfaces

MOZHGAN (NORA) ENTEKHABI and KIRK E. LANCASTER

A remark on the Noetherian property of power series rings

BYUNG GYUN KANG and PHAN THANH TOAN

Curves with prescribed intersection with boundary divisors in moduli spaces of curves

\section{XIAO-LEI LIU}

Virtual rational Betti numbers of nilpotent-by-abelian groups

BEHROOZ MiRZAII and FATEMEH Y. MOKARI

A planar Sobolev extension theorem for piecewise linear homeomorphisms 405

EMANUELA RADICI

A combinatorial approach to Voiculescu's bi-free partial transforms

PAUL SKOUFRANIS

Vector bundle valued differential forms on $\mathbb{N} Q$-manifolds

LUCA VitAGLiANO

Discriminants and the monoid of quadratic rings 